\title{
Use of the Functioning Assessment Short Test (FAST) in defining functional recovery in bipolar I disorder. Post-hoc analyses of long-term studies of aripiprazole once monthly as maintenance treatment
}

This article was published in the following Dove Press journal:

Neuropsychiatric Disease and Treatment

Jessica Madera'

Pedro Such ${ }^{2}$

Peter Zhang'

Ross A Baker'

Iria Grande ${ }^{3}$

'Medical Affairs, Otsuka Pharmaceutical Development and Commercialization Inc, Princeton, NJ, USA; ${ }^{2}$ Medical Affairs, $\mathrm{H}$. Lundbeck A/S, Valby, Denmark; ${ }^{3}$ Bipolar and Depression Disorders Unit, Hospital Clinic, Institute of Neurosciences, University of Barcelona, IDIBAPS, CIBERSAM, Barcelona, Catalonia, Spain
Correspondence: Iria Grande Bipolar and Depression Disorders Unit, Clinical Institute of Neurosciences, Hospital Clinic, University of Barcelona, IDIBAPS, CIBERSAM, Villarroel I70, Barcelona 08036, Spain

Tel +34932275400 ext 3130

$\mathrm{Fax}+34932279228$

Email igrande@clinic.cat
Purpose: There is growing agreement that definitions of "recovery" in bipolar-I disorder (BP-I) should include functional outcomes beyond sustained symptomatic remission. In this post-hoc analysis, we assessed functional recovery rates according to the validated Functioning Assessment Short Test (FAST) in participants with BP-I after 52 weeks of maintenance treatment with aripiprazole once monthly (AOM).

Patients and methods: Rates of functional recovery with AOM 400 were investigated in two 52-week studies. NCT01567527 was a placebo-controlled, double-blind, randomized-withdrawal study and NCT01710709 was an open-label study. Functional recovery, assessed at the end of the respective maintenance phases, was defined as a total FAST score of $\leq 11$ for 8 consecutive weeks. Results: Post-hoc analyses included 229 patients from the randomized-withdrawal study (AOM $400 \mathrm{n}=116$; placebo $\mathrm{n}=113$ ). The open-label study included 402 patients (including 321 de novo patients and 81 rollover patients who had completed the randomized-withdrawal study). In the randomized-withdrawal study, functional recovery was achieved by $30.2 \%$ $(n=35)$ of the AOM 400 group compared with $24.8 \%(n=28)$ in the placebo group. The difference was not statistically significant $(p=0.39)$. In the open-label study, $36 \%(\mathrm{n}=116)$ of de novo patients and $43 \%(n=35)$ of rollover patients had functionally recovered after 52 weeks of AOM 400 treatment.

Conclusion: These data highlight the utility of a sustained FAST total score of $\leq 11$ as a definition of recovery and emphasize the possibility of achieving this ambitious treatment goal with effective long-term treatment.

Keywords: bipolar disorder, aripiprazole, long-acting injectable, maintenance, functioning, recovery

\section{Plain language summary}

Functional recovery is beginning to be considered equally as important as symptomatic recovery in patients with bipolar I disorder (BP-I). We present post-hoc analyses of two studies in which we assessed functional recovery rates according to the validated Functioning Assessment Short Test (FAST) in participants with BP-I after 52 weeks of maintenance treatment with aripiprazole $400 \mathrm{mg}$ once monthly (AOM). To our knowledge, this is the first analysis of clinical trials to use the FAST scale as a definition of functional recovery, and we show that $30-43 \%$ of patients achieved functional recovery after 52 weeks' maintenance treatment with AOM. 


\section{Introduction}

While many people living with bipolar disorders regain psychosocial functioning upon symptomatic remission, the majority suffer persistent functional difficulties, often despite adequate control of their core affective symptoms. Such functional deficits include problems in their ability to work, study, live independently, maintain interpersonal relationships and participate in recreational activities. ${ }^{1}$ Mood stabilizers and/or atypical antipsychotics are well accepted as the mainstays of bipolar-I disorder (BP-I) treatment. ${ }^{2-4}$ Compared with their oral counterparts, longacting injectable (LAI) atypical antipsychotic formulations allow for better adherence with more consistent dosing ${ }^{2,4}$ and have recently shown to be more effective in preventing hospitalization of BP-I patients due to mental or physical illness. ${ }^{5}$ Aripiprazole $400 \mathrm{mg}$ once monthly (AOM 400) is an LAI approved by the US Food and Drug Administration as maintenance monotherapy treatment for BP-I. ${ }^{6}$ Results from recent placebo-controlled ${ }^{7}$ and open-label ${ }^{8}$ studies show that maintenance treatment with AOM 400 delays the time to mood episode recurrence and is safe and welltolerated.

There is growing agreement that definitions of "functional recovery" in bipolar disorders should include functional outcomes beyond sustained symptomatic remission. ${ }^{9}$ Both studies of AOM 400 as maintenance treatment used the Functioning Assessment Short Test (FAST), which was developed as a short simple interview-administered instrument for use in patients with psychiatric disorders, and especially bipolar disorders. ${ }^{10}$ The FAST has been shown to have strong psychometric properties and is able to detect differences between euthymic and acute patients with bipolar disorder. ${ }^{10}$ We have previously reported maintenance of improvement in FAST scores over 52 weeks in the AOM group of the placebo-controlled study. ${ }^{11}$ Taking into account the FAST cut off scores proposed by Rosa et al, ${ }^{10}$ we defined recovery as a FAST total score $\leq 11$ for $\geq 8$ consecutive weeks and assessed the rates of functional recovery in participants with BP-I after long-term (52-week) treatment with AOM 400.

\section{Materials and methods}

The efficacy and safety of AOM 400, given every 4 weeks, as maintenance treatment of BP-I was investigated in two, 52-week studies, the full methodologic details of which have been previously published:
1. A placebo-controlled, double-blind, randomizedwithdrawal study (NCT01567527) conducted in 103 sites in 7 countries. ${ }^{7}$

2. An open-label, multicenter study (NCT01710709) conducted in 149 sites in 10 countries. $^{8}$

Both studies ${ }^{7,8}$ were conducted in compliance with the Declaration of Helsinki and International Conference on Harmonization Good Clinical Practice guidelines for conducting, recording and reporting trials, as well as for archiving essential documents. Consistent with ethical principles for the protection of human research subjects, no trial procedures were performed on trial candidates until written consent had been obtained from them. The informed consent form, protocol and amendments for this trial were submitted to and approved by the institutional review board (IRB) or independent ethics committee (IEC) for each respective trial site or country (Table S1).

\section{Study design}

Study designs are summarized in Figure 1. Briefly, in the randomized-withdrawal study, participants completed oral aripiprazole conversion and stabilization phases if needed, followed by a single-blind AOM 400 stabilization phase. Those meeting stability criteria (outpatient status, Young Mania Rating Scale [YMRS] total score $\leq 12$, Montgomery-Asberg Depression Rating Scale [MADRS] total score $\leq 12$ and no active suicidality) were randomized to double-blind treatment with $\mathrm{AOM}$ 400 or placebo for 52 weeks.

The open-label study had two protocols, depending on whether the participants were de novo or had rolled-over from the randomized-withdrawal study. Whereas rollover participants began the 52-week, open-label AOM 400 maintenance phase immediately after completing the prior double-blind maintenance phase (AOM 400 or placebo), de novo participants entered a 4- to 12-week oral aripiprazole stabilization phase before entering the open-label maintenance phase. If de novo participants were receiving a non aripiprazole antipsychotic medication before enrollment, a 4- to 6week oral aripiprazole cross-titration phase was implemented before the oral aripiprazole stabilization phase.

\section{Participants}

Both studies enrolled outpatients (18-65 years) who had a clinical diagnosis of BP-I (DSM, 1994), and who were further verified by the Mini-International Neuropsychiatric Interview. 
A

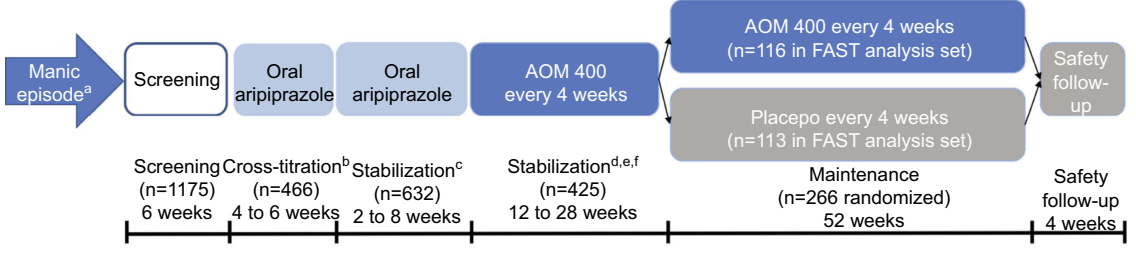

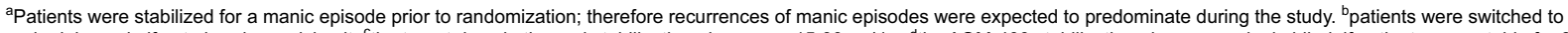
oral aripiprazole if not already receiving it. ${ }^{c}$ the target dose in the oral stabilization phase was $15-30 \mathrm{mg} /$ day. ${ }^{d}$ the AOM- 400 stabilization phase was single blind; if patients were stable for 8 consecutive weeks, they progressed to double-blind, randomized maintenace treatement. ${ }^{e}$ oral aripiprazole continued for 14 days following the first AOM 400 injection the dose of AOM

B could be changed to $300 \mathrm{mg}$ for tolerability.

$\mathbf{B}$

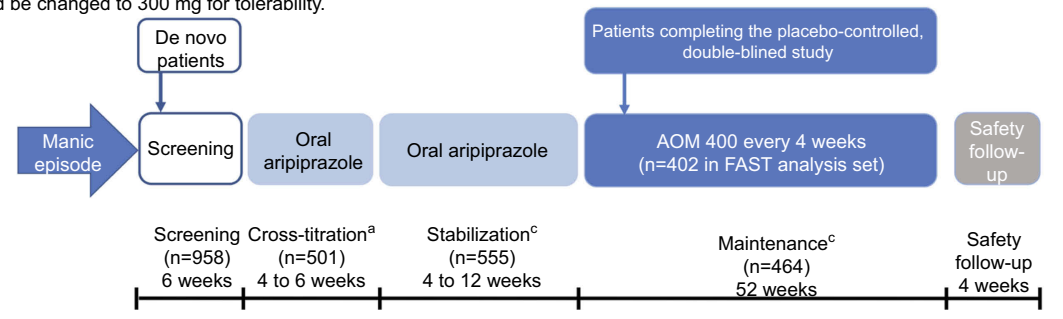

${ }^{a}$ During the cross-titration phase, patients were switched to oral aripiprzole if not already receiving it. ${ }^{b}$ the target dose in the stabilization phase was $15-30 \mathrm{mg} / \mathrm{day}$; if the patients was stable at one biweekly vists, they progressed to the AOM 400 stabilization phase. ${ }^{\circ}$ patients who had first participated in the randomized withdrawal study (AOM 400 And placebo, $n=81$ ) were subesquently enrolled in the open-label study entered directly into the maintenance phase of the open-label study. Together with the de nono patients entering from the stabilization phase $(n=321)$ this summed up to 402 patients in maintenance phase. Dose reductions to $300 \mathrm{mg}$ were permitted for tolerbility during the maintenance phase.

Figure I Study design. (A) Randomized-withdrawal study (Calabrese et al, 2017) ${ }^{7}$, (B) open-label maintenance study (Calabrese et al, 2018a). ${ }^{8}$ Abbreviation: AOM 400, Aripiprazole 400mg once-monthly.

Participants included in the open-label study were rolled over from the double-blind study or were AOM 400 treatmentnaive and enrolled de novo.

All participants in the randomized-withdrawal study and new participants recruited to the open-label study were eligible for the trial if they had experienced $\geq 1$ previous manic or mixed episode with manic symptoms of sufficient severity to require hospitalization, treatment with a mood stabilizer, or treatment with an antipsychotic agent. Study entry criteria were similar, except that the randomized-withdrawal study required participants to have a YMRS score $>20$ and excluded participants with a mixed or depressive episode, and the open-label study had no YMRS criterion and only excluded participants with a depressive episode. The open-label study also included rollover participants, who had completed the maintenance phase of the randomized-withdrawal study (AOM 400 or placebo) without recurrence of a mood episode. Participants previously on placebo had prior exposure to AOM 400 due to the 12- to 28-week AOM 400 stabilization phase.

\section{Analysis of functional recovery}

These analyses included all participants (both studies) who received maintenance study treatment and had $\geq 1$ post baseline FAST assessment. Functioning was assessed using the FAST (Table S2), where trained investigators ranked the participant's level of difficulty from 0 (no difficulty) to 3 (severe difficulty). Domains are based on grouping of the 24 individual items: autonomy (4 items), occupational functioning (5 items), cognitive functioning ( 5 items), financial issues (2 items), interpersonal relationships (6 items) and leisure time ( 2 items). The FAST total score (range 0-72) is calculated as the sum of each of the 24 item scores, with higher scores representing worse function. ${ }^{10}$ Any one missing score led to a missing total score. In addition, our definition included a minimum duration of 8 consecutive weeks to ensure that transient fluctuations were not designated as recovery. ${ }^{12}$

Functional recovery was thus defined post-hoc as a FAST total score of $\leq 11$ for 8 consecutive weeks. FAST total and domain scores (LOCF) were summarized at baseline and Week 52 of the respective maintenance phases using mean and SD for 1) all participants included in the analyses of FAST data and 2) those participants who met criteria for functional recovery. Between-group differences were derived from an ANOVA model with treatment and region as baseline factors.

\section{Results}

\section{Patient disposition and baseline characteristics}

Of the 266 participants entered into the randomizedwithdrawal phase of the placebo-controlled study, 116 received AOM 400 and 113 received placebo and had 
$\geq 1$ post-baseline FAST assessment. In the open-label study, 402 of the original 464 participants entering the maintenance phase had $\geq 1$ post baseline FAST assessment (321 de novo participants, 81 rollover participants). Overall $52 / 81$ of the rollover participants had already received treatment with AOM 400 for up to 52 weeks at baseline in the placebo-controlled, doubleblind study (for these participants total treatment duration, therefore, ranged between 52 and 80 weeks).

Baseline characteristics for the full populations of the two studies have been previously published. In brief, $57.5 \%$ of participants $(n=266)$ in the randomized-withdrawal study were female, the mean \pm SD age was $40.6 \pm 11.0$ years and age at first manic episode was $25.0 \pm 10.1$ years; participants had $3.5 \pm$ 4.0 prior hospitalizations for a mood episode. The mean YMRS total score was $2.8 \pm 3.3$, MADRS score was $2.7 \pm 3.4$ and FAST score was $15.4 \pm 12.7$ (Phase D baseline). ${ }^{7}$ For the open-label study, $57.8 \%$ of participants $(n=464)$ were female, the mean age was $41.1 \pm 11.8$ years and age at first BP-I diagnosis was $29.1 \pm 11.7$ years. The mean YMRS total score was 2.3 \pm 2.9 and MADRS score was $3.2 \pm 3.2 .^{8}$

\section{Rates of functional recovery}

During the maintenance phase of the placebo-controlled, double-blind study, $30.2 \%$ of participants (35/116) receiving AOM 400 and $24.8 \%$ of participants (28/113) receiving placebo achieved FAST recovery. Recovery rates were not statistically significant between AOM 400 and placebo groups $(p=0.3944$ [Cochrane-Mantel-Haenszel General Association Test
Controlling Region]). Of the participants who met recovery criteria in the double-blind phase, $33(\mathrm{n}=23$ previously treated with $\mathrm{AOM} 400$ and $n=10$ previously treated with placebo) agreed to "roll-over" into the subsequent open-label study.

In the open-label study, functional recovery as measured by FAST after 52 weeks of treatment was achieved by $36 \%$ of de novo participants $(\mathrm{n}=116)$ (Figure 2). Overall, $43 \%$ of rollover participants $(35 / 81)$ met the criteria for functional recovery. This included 20 participants who had previously received AOM 400 and met criteria for functional recovery in the double-blind study and who remained recovered after completing the following openlabel study (ie, they remained recovered into their second year). An additional 5 participants had not met criteria for functional recovery with AOM 400 during the placebocontrolled study but achieved functional recovery in the open-label study, while 3 participants were considered to meet functional recovery criteria in the placebo-controlled study but not in the open-label study. Of the participants previously on placebo, 10 met criteria for functional recovery with open-label AOM 400, including 8 who met criteria for recovery in both studies.

\section{FAST scores}

In the randomized-withdrawal study, FAST total scores were generally maintained in the group of participants who received AOM $400($ mean \pm SD score of $15.92 \pm$ 13.19 at baseline and $16.59 \pm 13.98$ at last visit) and were worsened in the placebo group (14.82 \pm 12.12 at baseline and $20.91 \pm 16.87$ at Week 52). The mean [95\% CI] treatment effect (AOM 400 vs placebo) of $-3.98[-7.52$,

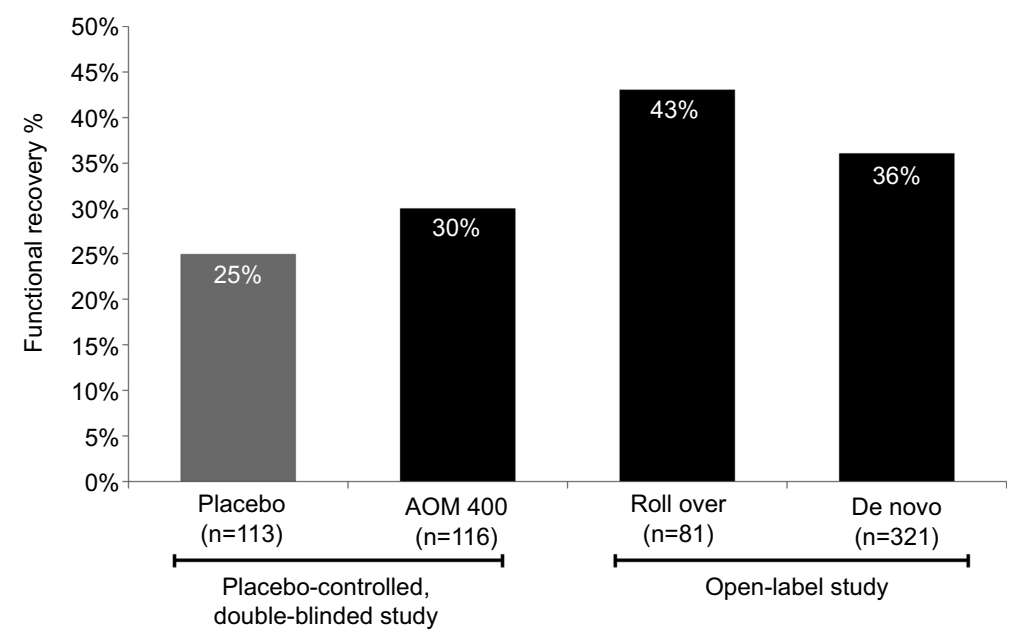

Figure 2 FAST recovery rates after long-term treatment with AOM 400 or placebo.

Abbreviations: FAST, Functioning Assessment Short Test; AOM 400, Aripiprazole 400 mg once monthly. 
$-0.44]$ at Week 52 was statistically significant $(p=0.028)$.

For those participants who met criteria for functional recovery, mean \pm SD FAST total scores numerically improved from $5.47 \pm 5.50$ at baseline to $3.51 \pm 3.62$ at Week 52 in the AOM 400 group and from $4.44 \pm 4.23$ at baseline to $2.75 \pm 2.86$ at Week 52 in the placebo group. Analyses by domain are provided in Table 1 .

In the open-label study, de novo participants significantly improved from a mean of $17.90 \pm 13.51$ at baseline to $14.02 \pm 12.02$ at the end of the 4- to 12 -week stabilization phase $(p<0.00001$, one-sided $\mathrm{Z}$ test). FAST total scores were then maintained during the 52-week maintenance phase (from $14.02 \pm 12.02$ to $13.98 \pm 13.05$ in de novo participants and from $12.89 \pm 12.22$ to $13.95 \pm 13.46$ in rollover participants). For those participants who met criteria for functional recovery, mean FAST total scores (baseline of maintenance phase/Week 52) were $4.09 \pm$ $4.00 / 3.59 \pm 3.39$ in the de novo group and $3.71 \pm 3.00 /$ $3.54 \pm 3.35$ in the rollover group.

\section{Discussion}

To the best of our knowledge, this is the first analysis of clinical trials to use FAST thresholds as a definition of functional recovery and highlight the scale's utility in understanding the effectiveness of an intervention. Our analyses of two long-term studies demonstrate that maintenance treatment with AOM 400 can help patients living with BP-I achieve long-term functional recovery. Overall, $30-43 \%$ of participants who received AOM 400 for at least 52 weeks achieved long-term functional recovery as determined by a FAST score of $\leq 11$ for at least 8 consecutive weeks.

Although the threshold for recovery used in these analyses was initially based on the FAST validation studies, which found that a score of $>11$ offers the best discriminant sensitivity and specificity, ${ }^{10}$ more recent studies support its use as a relatively conservative definition. According to Bonnin et al, euthymic BP-I outpatients in this category "present good functioning in all areas, they live independently, they work and they have a meaningful social engagement". ${ }^{13}$ In terms of recovery rates, our exploratory analyses of the randomized-withdrawal study did not show statistical separation from placebo. This is likely due, in part, to a survivor effect of the study design, where participants who initially had marked functional improvement and then stabilization (over 12-28 weeks) were able to remain functionally recovered, even when later randomized to placebo. Nevertheless, as previously reported, participants randomized to placebo showed a significant worsening in FAST scores relative to AOM and a higher risk of relapse. ${ }^{11}$

Overall, at least $57 \%$ of the participants who met criteria for functional recovery with AOM 400 maintenance treatment during the placebo-controlled study (and $87 \%$ of those who also chose to "roll over" to open-label treatment) remained recovered after completing the subsequent open-label study (ie, after 2 years of stable treatment). Of note, a small proportion of participants did not meet functional recovery criteria with active maintenance treatment during the first year, but did during the second year, thus supporting the idea that functional recovery takes longer to achieve than symptom recovery. ${ }^{14}$ Here, it is important to acknowledge that the entire rollover subgroup was highly enriched for patients who responded to and tolerated AOM treatment (during the stabilization phase for placebo patients and during the stabilization plus maintenance phases for AOM 400 patients). Indeed, due to the enriched discontinuation study designs, the generalizability of all results presented herein is limited to patients experiencing a manic episode and stabilized on AOM 400.

In terms of the maintenance effect (in both studies), there were only small changes in domain scores, with all functional domains remaining relatively stable with $\mathrm{AOM}$ 400 treatment. Likewise, all FAST domains appeared to remain similarly stable in the subgroup of participants who had functionally recovered. Subgroup analyses of the functionally "recovered" participants showed that mean FAST total scores were already $<6$ at baseline of the maintenance phase, which is similar to a control group of participants without bipolar disorder (mean of 5.93). ${ }^{15}$ The rates of functional recovery seen with long-term AOM treatment are in line with or slightly higher than those previously reported after 52 weeks of olanzapine treatment. ${ }^{16}$ However, it should also be noted that the olanzapine study defined functional recovery using a combination of the psychosocial functioning sub scale of the SF-36 and work status and disability support measures - the comparability of which with the validated FAST scale is unknown.

Limitations of this study include the post-hoc nature of the recovery rate analyses and the lack of a blinded comparator in the open-label study. Whereas the minimum duration of 8 consecutive weeks could be considered relatively short, ${ }^{9}$ we based our definition to be consistent with the recommendations of the International Society for 
Table I FAST scores at baseline and Week 52 of each study (LOCF)

\begin{tabular}{|c|c|c|c|c|c|}
\hline & \multicolumn{3}{|c|}{ Randomized-withdrawal study } & \multicolumn{2}{|c|}{$\begin{array}{l}\text { Open-label AOM } 400 \\
\text { maintenance study }\end{array}$} \\
\hline & AOM 400 & Placebo & Treatment effect (AOM vs placebo); LS mean $[95 \% \mathrm{Cl}]$ & De novo & Rollover \\
\hline \multicolumn{6}{|l|}{ Autonomy score } \\
\hline \multicolumn{6}{|l|}{ Total population } \\
\hline Baseline; mean (SD) & $\mathrm{I} .47(2.07)$ & $1.35(2.10)$ & $-0.67[-1.35,0.02]$ & $1.23(1.89)$ & $1.19(2.07)$ \\
\hline Week 52; mean (SD) & $1.72(2.49)$ & $2.43(3.19)$ & $P=0.055$ & $1.19(1.46)$ & $1.46(2.27)$ \\
\hline \multicolumn{6}{|l|}{ Recovery population } \\
\hline Baseline; mean (SD) & $0.29(0.63)$ & $0.04(0.19)$ & $0.01[-0.23,0.24]$ & $0.28(0.79)$ & $0.26(0.78)$ \\
\hline Week 52; mean (SD) & $0.17(0.45)$ & $0.11(0.42)$ & $P=0.958$ & $0.24(0.60)$ & $0.34(0.94)$ \\
\hline \multicolumn{6}{|c|}{ Occupational functioning score } \\
\hline \multicolumn{6}{|l|}{ Total population } \\
\hline Baseline; mean (SD) & $5.73(4.91)$ & $5.69(5.35)$ & $-0.82[-1.88,0.24]$ & 4.69 (4.69) & $4.42(5.18)$ \\
\hline Week 52; mean (SD) & $5.29(4.91)$ & $6.39(5.54)$ & $P=0.128$ & 4.50 (4.69) & $5.05(5.26)$ \\
\hline \multicolumn{6}{|l|}{ Recovery population } \\
\hline Baseline; mean (SD) & $2.26(2.74)$ & $1.56(1.89)$ & $0.24[-0.63,1.11]$ & $1.15(1.85)$ & $0.97(1.32)$ \\
\hline Week 52; mean (SD) & $1.14(1.65)$ & $0.93(1.59)$ & $P=0.582$ & $1.04(1.83)$ & $0.91(1.38)$ \\
\hline \multicolumn{6}{|c|}{ Cognitive functioning score } \\
\hline \multicolumn{6}{|l|}{ Total population } \\
\hline Baseline; mean (SD) & $3.28(3.38)$ & $2.91(3.18)$ & $-0.56(-1.44,0.32]$ & $2.97(2.99)$ & $2.69(3.20)$ \\
\hline Week 52; mean (SD) & $3.64(3.62)$ & $4.21(4.06)$ & $P=0.212$ & $2.00(3.14)$ & $2.86(3.42)$ \\
\hline \multicolumn{6}{|l|}{ Recovery population } \\
\hline Baseline; mean (SD) & $0.97(1.57)$ & $0.96(1.29)$ & $0.32[-0.12,0.76]$ & $1.14(1.38)$ & $0.97(1.67)$ \\
\hline Week 52; mean (SD) & $0.66(1.21)$ & $0.29(0.66)$ & $P=0.155$ & $0.96(1.42)$ & $0.86(1.50)$ \\
\hline \multicolumn{6}{|l|}{ Financial issues score } \\
\hline \multicolumn{6}{|l|}{ Total population } \\
\hline Baseline; mean (SD) & $1.20(1.66)$ & $1.17(1.48)$ & $-0.35[-0.74,0.04]$ & 1.17 (1.59) & $0.93(1.40)$ \\
\hline Week 52; mean (SD) & $1.16(1.53)$ & $1.57(1.72)$ & $P=0.075$ & $1.09(1.58)$ & $1.09(1.49)$ \\
\hline \multicolumn{6}{|l|}{ Recovery population } \\
\hline Baseline; mean (SD) & $0.32(0.68)$ & $0.57(1.23)$ & $-0.06[-0.49,0.36]$ & $0.40(0.90)$ & $0.31(0.76)$ \\
\hline Week 52; mean (SD) & $0.29(0.83)$ & $0.43(0.88)$ & $P=0.777$ & $0.4 I(0.95)$ & $0.31(0.76)$ \\
\hline \multicolumn{6}{|c|}{ Interpersonal relationships score } \\
\hline \multicolumn{6}{|l|}{ Total population } \\
\hline Baseline; mean (SD) & $2.84(3.50)$ & $2.45(2.87)$ & $-1.32[-2.32,-0.31]$ & $2.75(3.33)$ & $2.52(3.38)$ \\
\hline Week 52; mean (SD) & $3.15(3.82)$ & $4.50(4.67)$ & $P=0.011$ & $2.71(3.63)$ & $2.33(3.62)$ \\
\hline \multicolumn{6}{|l|}{ Recovery population } \\
\hline Baseline; mean (SD) & $1.15(1.62)$ & $0.71(1.01)$ & $0.17[-0.37,0.70]$ & $0.67(1.09)$ & $0.66(1.21)$ \\
\hline Week 52; mean (SD) & $0.77(1.26)$ & $0.46(0.79)$ & $P=0.537$ & $0.50(1.01)$ & $0.63(1.03)$ \\
\hline \multicolumn{6}{|l|}{ Leisure time score } \\
\hline \multicolumn{6}{|l|}{ Total population } \\
\hline Baseline; mean (SD) & $\mathrm{I} .40(\mathrm{I} .57)$ & $1.18(1.28)$ & $-0.19(-0.58,0.20)$ & $1.27(1.44)$ & $1.14(1.40)$ \\
\hline Week 52; mean (SD) & $1.62(1.65)$ & $1.81(1.76)$ & $P=0.339$ & $1.45(1.62)$ & $1.16(1.45)$ \\
\hline \multicolumn{6}{|l|}{ Recovery population } \\
\hline Baseline; mean (SD) & $0.47(1.08)$ & $0.50(0.64)$ & $-0.00[-0.38,0.38]$ & $0.46(0.78)$ & $0.54(0.89)$ \\
\hline Week 52; mean (SD) & $0.49(0.92)$ & $0.54(0.69)$ & $P=0.992$ & $0.44(0.86)$ & $0.49(0.82)$ \\
\hline
\end{tabular}


Bipolar Disorders for symptomatic recovery. ${ }^{12}$ While LOCF analyses can be criticized, we used the same methods of imputation as per the primary study analyses. ${ }^{7,8}$ Although the roll over group provided important insights into the longevity and time course of functional recovery, it is important that almost half $(47.6 \%)$ of the participants who met criteria for functional recovery in the doubleblind phase did not choose to "roll over" to the openlabel trial. Finally, it has been recently suggested that euthymic patients can be categorized into three main functional types, low-, intermediate- and high-functioning, and that the low- and intermediate-functioning types have higher subthreshold depressive/manic symptoms and worse cognition, particularly in terms of processing speed. ${ }^{17}$ Future studies including patients with depression as well as cognitive assessments may lend further clarity to the predictors of long-term functional recovery with AOM 400

\section{Conclusion}

Functional recovery is beginning to be considered equally as important as symptomatic recovery, since key goals for patients and relatives are to fulfill role expectations at work/school and home and to maintain good relationships. ${ }^{18-20}$ Almost all individuals with bipolar disorders require maintenance treatment to prevent subsequent episodes, reduce residual symptoms and restore functioning. ${ }^{4}$ The results of this study demonstrate the utility of a FAST total score of $\leq 11$ for 8 consecutive weeks as a definition of functional recovery in BP-I and highlight the possibility of achieving this ambitious treatment goal with effective treatment.

\section{Ethics approval and informed consent}

Both studies were conducted in compliance with the International Conference of Harmonization and Good Clinical Practice consolidated guideline. ${ }^{7,8}$ Protocols were approved by an institutional review board or independent ethics committee, as appropriate. Informed consent was obtained from all participants or their legal representatives as necessary.

\section{Abbreviations}

AOM, aripiprazole once monthly; BP-I, bipolar I disorder; FAST, Functioning Assessment Short Test; LAI, long-acting injectable; MADRS, Montgomery-Asberg Depression Rating Scale; YMRS, Young Mania Rating Scale.

\section{Data sharing statement}

Otsuka and H. Lundbeck are committed to sharing data in accordance with the European Federation of Pharmaceutical Industries and Associations (EFPIA)/Pharmaceutical Research and Manufacturers of America (PhRMA) principles for responsible sharing of clinical trial data guidelines and as required by applicable legislation. Legitimate research requests will be considered. For inquiries on availability of data of interest, researchers should contact Otsuka (DT-inquiry@otsuka.jp). Please visit https://clinical-trials. otsuka.com/For-Researchers.aspx for further details.

\section{Acknowledgments}

We wish to thank Anita Chadha-Patel (ACP Clinical Communications Ltd, funded by Otsuka Pharmaceutical Development and Commercialization Inc. and $\mathrm{H}$. Lundbeck $\mathrm{A} / \mathrm{S}$ ) for support in the preparation, revisions and editing of this article. We also thank all investigators and participants in the two long-term studies. This work has been sponsored by Otsuka Pharmaceutical Development \& Commercialization, Inc. and $\mathrm{H}$. Lundbeck A/S. The sponsors were involved in the design and conduct in both studies.

\section{Author contributions}

All authors contributed to data analysis, drafting and revising the article, gave final approval of the version to be published, and agree to be accountable for all aspects of the work.

\section{Disclosure}

Jessica Madera, Peter Zhang and Ross A Baker are employed by Otsuka Pharmaceutical Development and Commercialization Inc. Pedro Such is employed by $\mathrm{H}$. Lundbeck A/S. Iria Grande reports consultancy for Otsuka Pharmaceutical Development and Commercialization Inc. and $\mathrm{H}$. Lundbeck A/S. Iria Grande also reports grants, personal fees, and non-financial support from the Spanish Ministry of Economy and Competitiveness, Instituto de Salud Carlos III. She received personal fees from AstraZeneca, Ferrer, Janssen Cilag, H. Lundbeck, and Lundbeck-Otsuka, during the conduct of the study and outside the submitted work. The authors report no other conflicts of interest in this work. 


\section{References}

1. Sanchez-Moreno J, Martinez-Aran A, Tabares-Seisdedos R, Torrent C, Vieta E, Ayuso-Mateos JL. Functioning and disability in bipolar disorder: an extensive review. Psychother Psychosom. 2009;78 (5):285-297. doi:10.1159/000228249

2. Chou YH, Chu PC, Wu SW, et al. A systemic review and experts' consensus for long-acting injectable antipsychotics in bipolar disorder. Clin Psychopharmacol Neurosci. 2015;13(2):121-128. doi:10.9758/ cpn.2015.13.2.121

3. Derry S, Moore RA. Atypical antipsychotics in bipolar disorder: systematic review of randomised trials. BMC Psychiatry. 2007;7:40. doi:10.1186/1471-244X-7-40

4. Vieta E, Berk M, Schulze TG, et al. Bipolar disorders. Nat Rev Dis Primers. 2018;4:18008. doi:10.1038/nrdp.2018.8

5. Lahteenvuo M, Tanskanen A, Taipale H, et al. Real-world effectiveness of pharmacologic treatments for the prevention of rehospitalization in a finnish nationwide cohort of patients with bipolar disorder. JAMA Psychiatry. 2018;75(4):347-355. doi:10.1001/jamapsychiatry.2017.4711

6. Torres-Llenza V, Lakshmin P, Lieberman DZ. Spotlight on once-monthly long-acting injectable aripiprazole and its potential as maintenance treatment for bipolar I disorder in adult patients. Neuropsychiatr Dis Treat. 2018;14:285-292. doi:10.2147/NDT.S129559

7. Calabrese JR, Sanchez R, Jin N, et al. Efficacy and safety of aripiprazole once-monthly in the maintenance treatment of bipolar I disorder: a doubleblind, placebo-controlled, 52-week randomized withdrawal study. J Clin Psychiatry. 2017;78(3):324-331. doi:10.4088/JCP.16m11201

8. Calabrese JR, Jin N, Johnson B, et al. Aripiprazole once-monthly as maintenance treatment for bipolar I disorder: a 52-week, multicenter, open-label study. Int J Bipolar Disord. 2018;6(1):14. doi:10.1186/ s40345-018-0122-z

9. Harvey PD. Defining and achieving recovery from bipolar disorder. $J$ Clin Psychiatry. 2006;67(Suppl 9):14-18; discussion 36-42.

10. Rosa AR, Sanchez-Moreno J, Martinez-Aran A, et al. Validity and reliability of the Functioning Assessment Short Test (FAST) in bipolar disorder. Clin Pract Epidemiol Ment Health. 2007;3:5. doi:10.1186/1745-0179-3-5
11. Calabrese JR, Sanchez R, Jin N, et al. Symptoms and functioning with aripiprazole once-monthly injection as maintenance treatment for bipolar I disorder. $J$ Affect Disord. 2018;227:649-656.

12. Tohen M, Frank E, Bowden CL, et al. The International Society for Bipolar Disorders (ISBD) task force report on the nomenclature of course and outcome in bipolar disorders. Bipolar Disord. 2009;11 (5):453-473. doi:10.1111/j.1399-5618.2009.00726.x

13. Bonnin CM, Martinez-Aran A, Reinares M, et al. Thresholds for severity, remission and recovery using the functioning assessment short test (FAST) in bipolar disorder. $J$ Affect Disord. 2018;240:5762. doi:10.1016/j.jad.2018.07.045

14. Gitlin MJ, Miklowitz DJ. The difficult lives of individuals with bipolar disorder: a review of functional outcomes and their implications for treatment. J Affect Disord. 2017;209:147-154. doi:10.1016/ j.jad.2016.11.021

15. Rosa AR, Bonnin CM, Vazquez GH, et al. Functional impairment in bipolar II disorder: is it as disabling as bipolar I? J Affect Disord. 2010;127(1-3):71-76. doi:10.1016/j.jad.2010.05.014

16. Chengappa KN, Hennen J, Baldessarini RJ, et al. Recovery and functional outcomes following olanzapine treatment for bipolar I mania. Bipolar Disord. 2005;7(1):68-76. doi:10.1111/j.13995618.2004.00171.x

17. Sole B, Bonnin CM, Jimenez E, et al. Heterogeneity of functional outcomes in patients with bipolar disorder: a cluster-analytic approach. Acta Psychiatr Scand. 2018;137(6):516-527. doi:10.1111/acps.12871

18. Dodd AL, Mezes B, Lobban F, Jones SH. Psychological mechanisms and the ups and downs of personal recovery in bipolar disorder. $\mathrm{Br} \mathrm{J}$ Clin Psychol. 2017;56(3):310-328. doi:10.1111/bjc.12140

19. Grande I, Goikolea JM, de Dios C, et al. Occupational disability in bipolar disorder: analysis of predictors of being on severe disablement benefit (PREBIS study data). Acta Psychiatr Scand. 2013;127 (5):403-411. doi:10.1111/acps.12003

20. Michalak EE, Yatham LN, Kolesar S, Lam RW. Bipolar disorder and quality of life: a patient-centered perspective. Qual Life Res. 2006;15 (1):25-37. doi:10.1007/s11136-005-0376-7 


\section{Supplementary materials}

Table SI The Functioning Assessment Short Test (FAST)

\section{Physician rates each item according to difficulty:}

\section{(0) None (1) mild (2) moderate (3) severe}

\section{AUTONOMY}

I. Taking responsibility for a household

2. Living on your own

3. Doing shopping

4. Taking care of yourself (physical aspects, hygiene)

OCCUPATIONAL FUNCTIONING

5. Holding down a paid job

6. Accomplishing tasks as quickly as necessary

7. Working in the field in which you were educated

8. Occupational earnings

9. Managing the expected workload

\section{COGNITIVE FUNCTIONING}

10. Ability to concentrate on a book, film

II. Ability to make mental calculations

12. Ability to solve a problem adequately

13. Ability to remember newly-learned names

14. Ability to learn new information
FINANCIAL ISSUES

15. Managing your own money

16. Spending money in a balanced way

INTERPERSONAL RELATIONSHIPS

17. Maintaining a friendship or friendships

18. Participating in social activities

19. Having good relationships with people close to you

20. Living together with your family

21. Having satisfactory sexual relationships

22. Being able to defend you interests

LEISURE TIME

23. Doing exercise or participating in sport

24. Having hobbies or personal interests

Note: Data from Rosa et al (2007). ${ }^{10}$ 


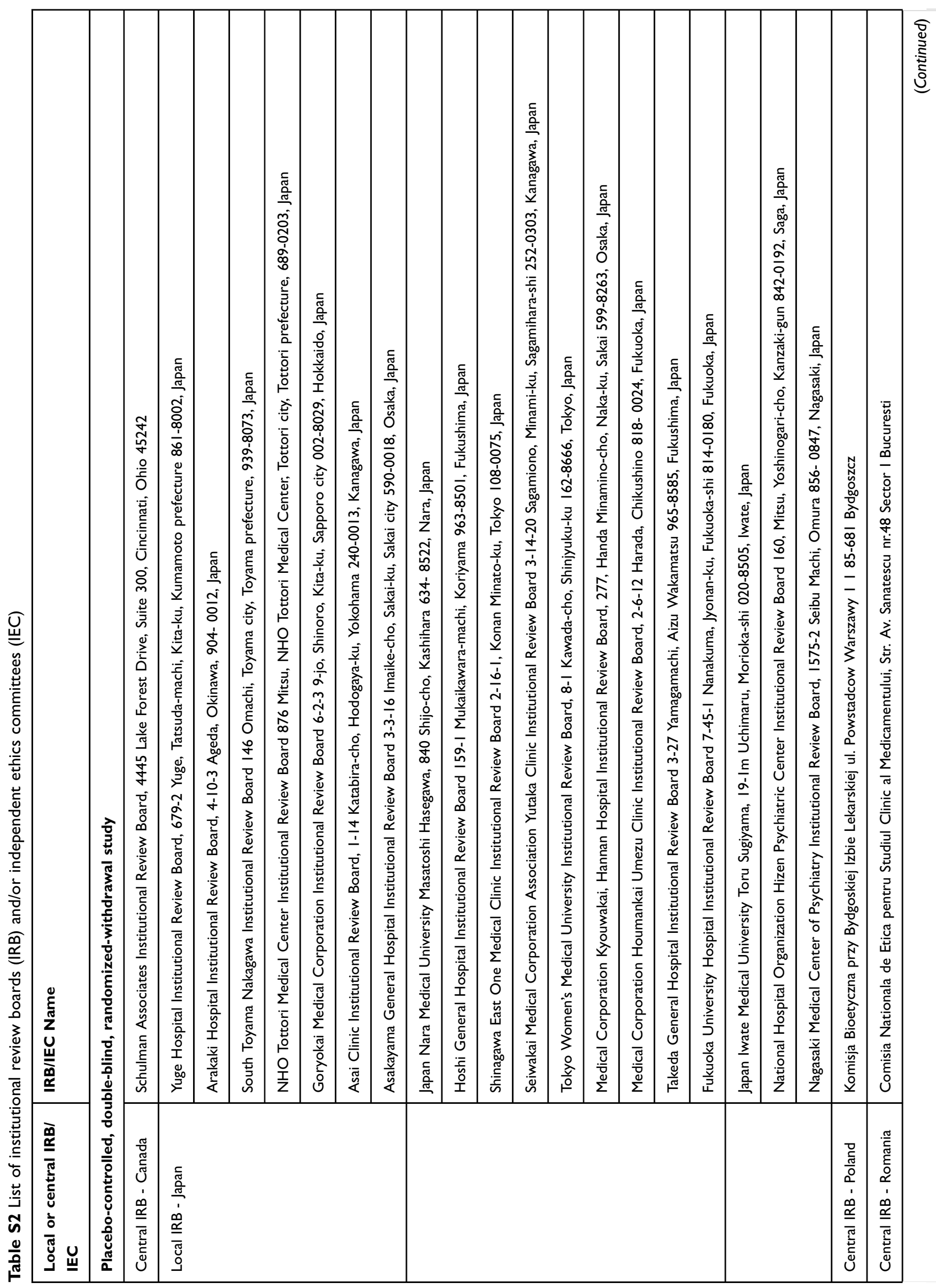




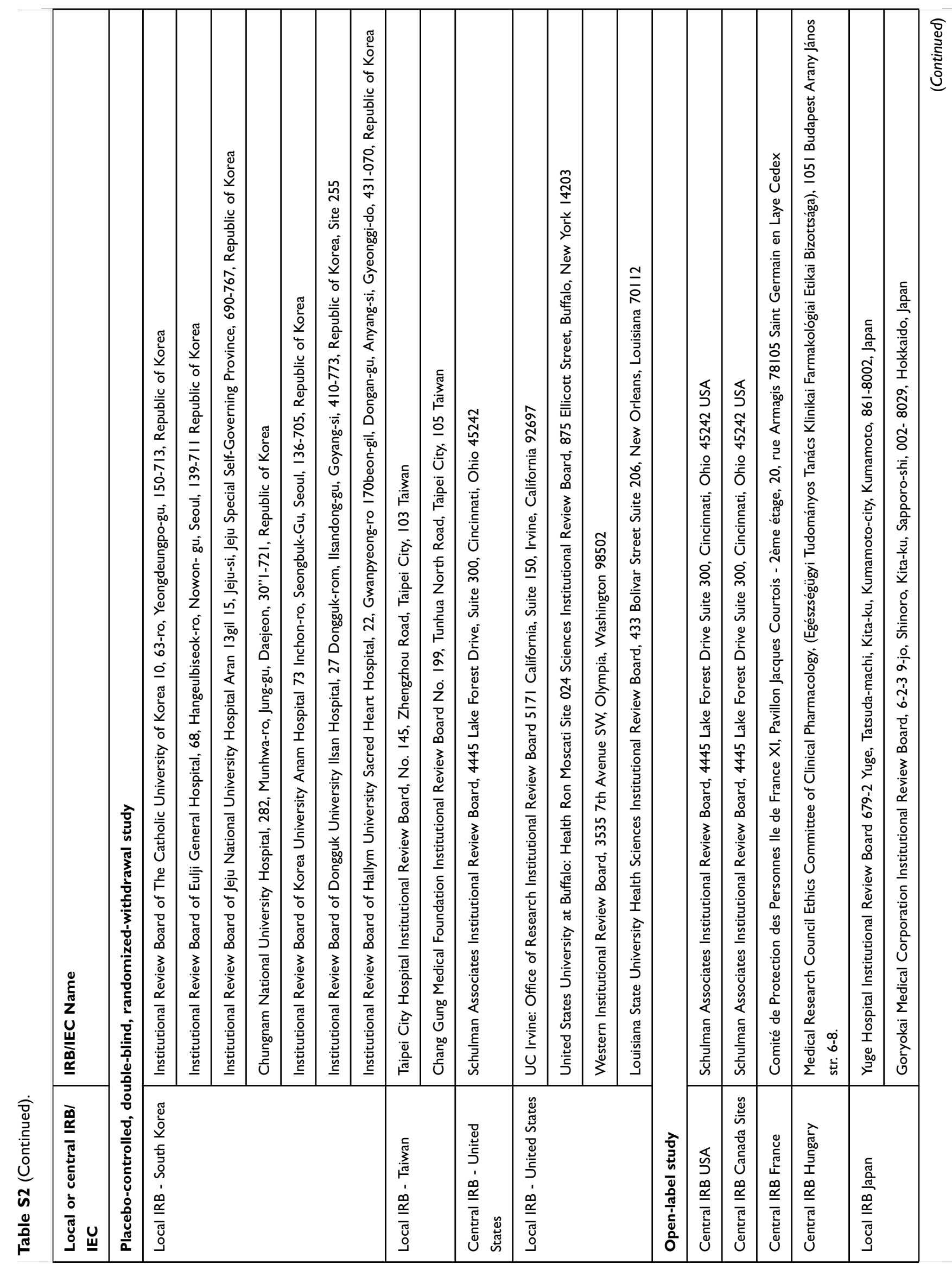




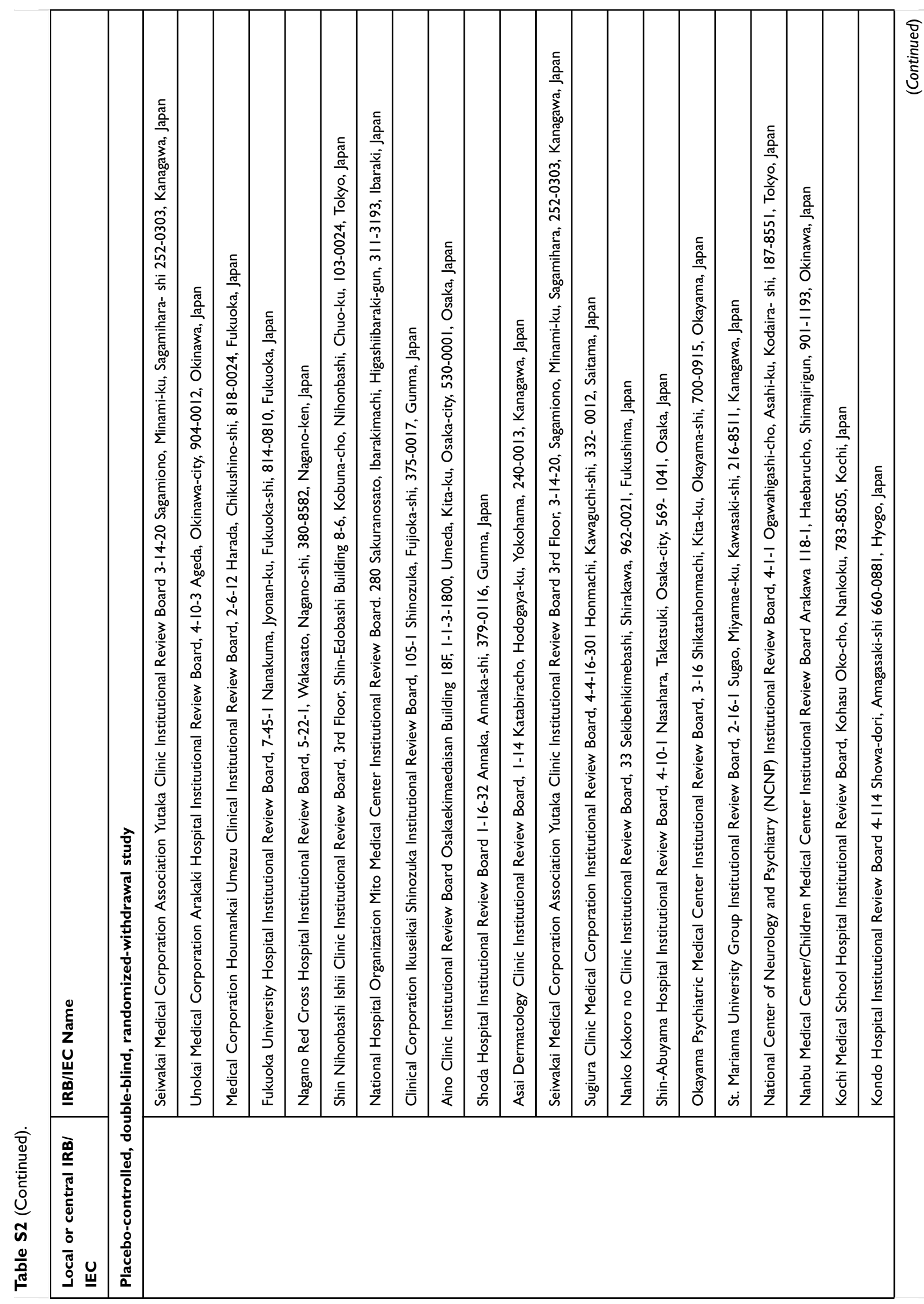




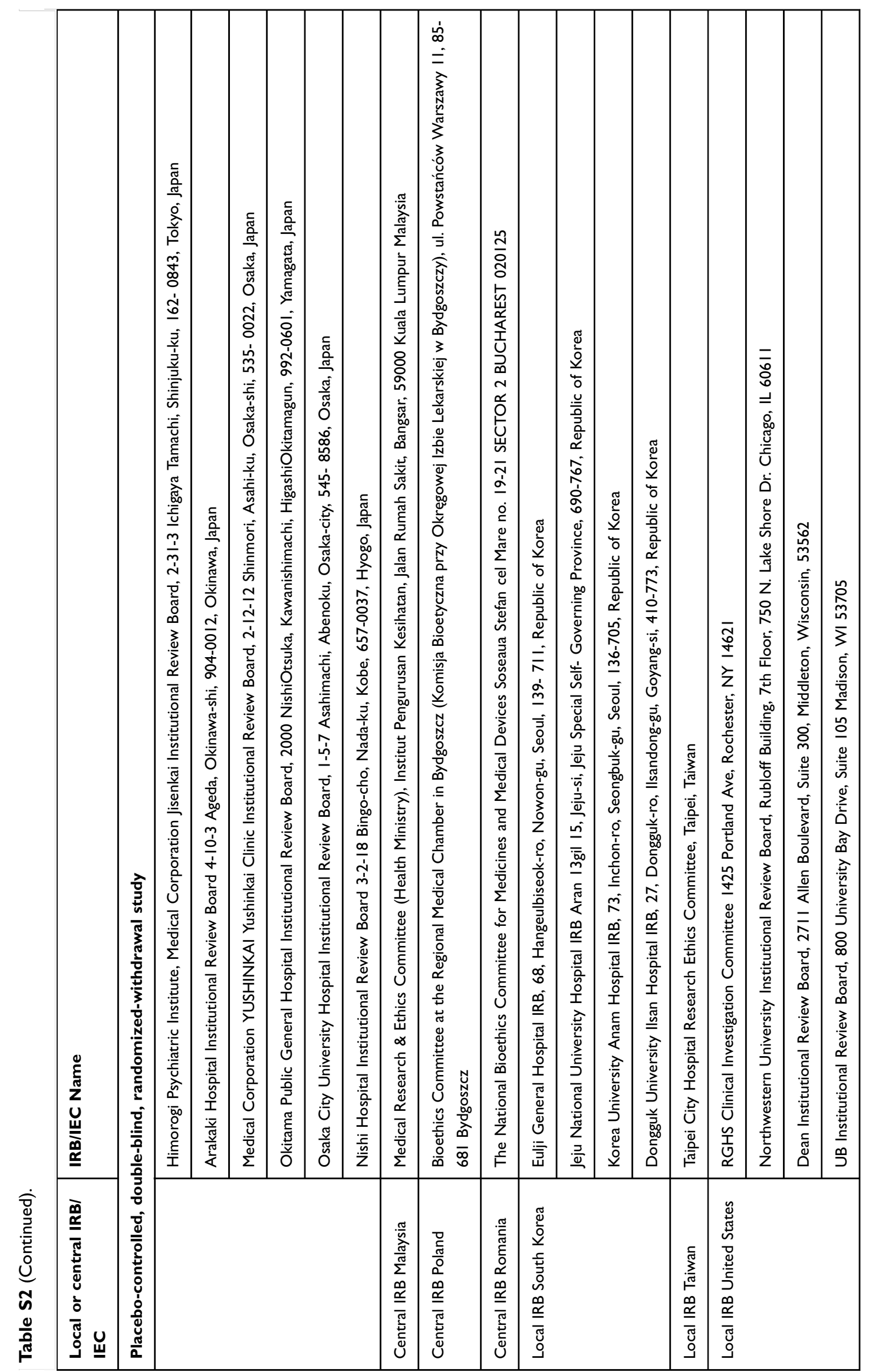




\section{Publish your work in this journal}

Neuropsychiatric Disease and Treatment is an international, peerreviewed journal of clinical therapeutics and pharmacology focusing on concise rapid reporting of clinical or pre-clinical studies on a range of neuropsychiatric and neurological disorders. This journal is indexed on PubMed Central, the 'PsycINFO' database and CAS, and is the official journal of The International Neuropsychiatric Association (INA). The manuscript management system is completely online and includes a very quick and fair peer-review system, which is all easy to use. Visit http://www.dovepress.com/testimonials.php to read real quotes from published authors. 